\title{
Elevated Pulse Pressure is Associated with Hemolysis, Proteinuria and Chronic Kidney Disease in Sickle Cell Disease
}

\author{
Enrico M. Novelli ${ }^{1 *}$, Mariana Hildesheim ${ }^{2}$, Caterina Rosano ${ }^{3}$, Rebecca \\ Vanderpool $^{4}$, Marc Simon ${ }^{4}$, Gregory J. Kato ${ }^{1}$, Mark T. Gladwin ${ }^{5}$ \\ 1. Vascular Medicine Institute and Division of Hematology/Oncology, University of Pittsburgh, Pittsburgh, \\ Pennsylvania, United States of America, 2. Critical Care Medicine Department, Clinical Center, National \\ Institutes of Health, Bethesda, Maryland, United States of America, 3. Department of Epidemiology, University \\ of Pittsburgh, Pittsburgh, Pennsylvania, United States of America, 4. Vascular Medicine Institute and Division \\ of Cardiology, University of Pittsburgh, Pittsburgh, Pennsylvania, United States of America, 5. Vascular \\ Medicine Institute, Division of Pulmonary, Allergy, Critical Care Medicine, University of Pittsburgh, Pittsburgh, \\ Pennsylvania, United States of America
}

*novellie@upmc.edu

\section{Abstract}

\section{OPEN ACCESS}

Citation: Novelli EM, Hildesheim M, Rosano C, Vanderpool R, Simon M, et al. (2014) Elevated Pulse Pressure is Associated with Hemolysis, Proteinuria and Chronic Kidney Disease in Sickle Cell Disease. PLoS ONE 9(12): e114309. doi:10. 1371/journal.pone.0114309

Editor: Dominique Guerrot, Rouen University Hospital, France

Received: July 21, 2014

Accepted: November 8, 2014

Published: December 5, 2014

This is an open-access article, free of all copyright, and may be freely reproduced, distributed, transmitted, modified, built upon, or otherwise used by anyone for any lawful purpose. The work is made available under the Creative Commons $\mathrm{CCO}$ public domain dedication.

Data Availability: The authors confirm that all data underlying the findings are fully available without restriction. All relevant data are within the paper.

Funding: EMN, MH, RV, MS, GJK and MTG are supported by the Institute for Transfusion Medicine and the Hemophilia Center of Western Pennsylvania. The funders had no role in study design, data collection and analysis, decision to publish, or preparation of the manuscript.

Competing Interests: The authors have declared that no competing interests exist.
A seeming paradox of sickle cell disease is that patients do not suffer from a high prevalence of systemic hypertension in spite of endothelial dysfunction, chronic inflammation and vasculopathy. However, some patients do develop systolic hypertension and increased pulse pressure, an increasingly recognized major cardiovascular risk factor in other populations. Hence, we hypothesized that pulse pressure, unlike other blood pressure parameters, is independently associated with markers of hemolytic anemia and cardiovascular risk in sickle cell disease. We analyzed the correlates of pulse pressure in patients $(n=661)$ enrolled in a multicenter international sickle cell trial. Markers of hemolysis were analyzed as independent variables and as a previously validated hemolytic index that includes multiple variables. We found that pulse pressure, not systolic, diastolic or mean arterial pressure, independently correlated with high reticulocyte count (beta $=$ 2.37, $p=0.02$ ) and high hemolytic index (beta $=1.53, p=0.002$ ) in patients with homozygous sickle cell disease in two multiple linear regression models which include the markers of hemolysis as independent variables or the hemolytic index, respectively. Pulse pressure was also independently associated with elevated serum creatinine (beta $=3.21, p=0.02$ ), and with proteinuria (beta $=2.52, p=$ 0.04). These results from the largest sickle cell disease cohort to date since the Cooperative Study of Sickle Cell Disease show that pulse pressure is independently associated with hemolysis, proteinuria and chronic kidney disease. We propose that high pulse pressure may be a risk factor for clinical complications 
of vascular dysfunction in sickle cell disease. Longitudinal and mechanistic studies should be conducted to confirm these hypotheses.

\section{Introduction}

Findings from the landmark Cooperative Study of Sickle Cell Disease (CSSCD) revealed that sickle cell disease (SCD) patients, particularly those affected by HbSS, have lower systolic and diastolic blood pressure values compared to age, sex and race-matched normative values.[1] This large study confirmed findings of prior smaller series showing lower baseline blood pressure values are characteristic of SCD patients. [2, $\underline{3}]$

Multiple hypotheses have been formulated over the following years to explain the paradox of a population affected by severe endotheliopathy and vasculopathy and accelerated organ damage, yet largely spared from systemic hypertension. Possible explanations have taken into consideration the role of anemia, resulting in increased cardiac output with compensatory decreased vascular resistance, hyposthenuria leading to sodium loss, decreased blood viscosity of oxygenated sickle blood at low hematocrit levels, and the role of compensatory increases in cyclooxygenase-2, endothelial nitric oxide synthase, placenta growth factor and other endothelial-derived factors.[4-7] Again, paradoxically, endothelial function studies in humans clearly show that many patients, especially those with higher rates of hemolytic anemia, exhibit impaired response to major endothelial vasodilators, such as nitric oxide. $[\underline{6}, \underline{8}, \underline{9}]$ More recent studies have also shown that when an appropriate control population is selected and potential confounders are accounted for statistically, the blood pressure difference between SCD patients and control subjects is attenuated.[5] Adjustment for the lower BMI of SCD patients as compared to that of control subjects has proved to be particularly important in evaluating blood pressure differences. $[\underline{5}, \underline{10}, \underline{11}]$ Another possibility is that systolic, diastolic and mean arterial pressure may not be the systemic pressure parameters most reflective of SCD vasculopathy. This hypothesis is supported by recent epidemiological evidence showing that systolic blood pressure rises as biomarkers of pulmonary hypertension and intravascular hemolysis increase, while diastolic blood pressure decreases as hemoglobin levels drop in SCD, leading to subgroups of patients developing systolic hypertension and increased pulse pressure.[12]

Elevated pulse pressure has recently emerged as an independent predictor of cardiovascular outcomes and prognostic factor for death in patients at high risk of cardiovascular morbidity and mortality such as aging patients and those affected by advanced chronic kidney disease. $[13,14]$ The main determinants of pulse pressure are the left ventricular ejection fraction, arterial stiffness, and the timing and intensity of the backward pressure wave reflections from the periphery of the vascular system.[15] From the hemodynamic factors that influence pulse pressure, 
two have been shown to independently predict cardiovascular risk: aortic stiffness, measured from the aortic pulse wave velocity, and early return of reflected waves to the heart, evaluated from pulse wave analysis.[15] Under physiological conditions in young subjects, the backward pressure wave returns from the distal arterial compartment during diastole, cause pulse pressure to be higher in peripheral than in central arteries, a phenomenon known as pulse pressure amplification.[15] In conditions where pulse wave velocity and arterial stiffness are increased, such as SCD,[16] the reflected wave occurs earlier affecting the central arteries during systole. As a result of this early wave reflection, aortic and ventricular pressures are increased during systole and aortic pressure is reduced during diastole,[15] leading to increased pulse pressure. The result of increased pulse pressure is greater vascular load on the heart, which can lead to myocardial hypertrophy and heart failure,[17] and end-organ damage in other vascular districts, including the kidney (reviewed in[18]) and the brain.[19]

Hemolysis in SCD causes endothelial dysfunction via multiple mechanisms, and both hemolysis and endothelial dysfunction may be independently linked to elevated pulse pressure. High plasma levels of free hemoglobin from hemolyzed $\mathrm{RBC}$ lead to nitric oxide depletion and reactive oxygen species formation which cause impaired vascular relaxation and increased arterial stiffness.[20] In addition, ischemia/reperfusion injury from acute episodes of vaso-occlusion and hemolysis also lead to the generation of pathologic reactive oxygen species responsible for endotheliopathy and endothelial inflammation.[21,22] The hemodynamic consequences of high baseline hemolysis and anemia are high cardiac output and decreased peripheral vascular resistance. The combination of increased arterial stiffness from endothelial dysfunction and a high cardiac output state from severe hemolysis and anemia are expected to elevate pulse pressure in SCD. We, therefore, hypothesized that hemolysis would be significantly associated with pulse pressure in patients with HbSS disease. In the study presented herein, we report the correlates of pulse pressure in the Treatment of Pulmonary Hypertension and Sickle Cell Disease with Sildenafil Therapy (walk-PHaSST) cohort.

\section{Methods}

\section{Study Design and Selection of Subjects}

The study population and design have been described in detail elsewhere.[23, 24] Overall, we recruited 720 subjects age 12 and over at steady state from nine different study sites in the United States and one site in the United Kingdom, 671 (93.2\%) of whom enrolled in the study. We analyzed 661 patients with available systolic and diastolic blood pressure measurements (98.5\% of enrolled subjects). 


\section{Ethics Statement}

Local institutional review boards (University of Pittsburgh IRB PRO07060076) approved the protocol and written informed consent was obtained and approved by the IRB (Clinicaltrials.gov identifier NCT00492531). This study is an analysis of data from the walk-PHaSST trial.

\section{Evaluation of Subjects}

All screening study subjects were evaluated by histories of clinical events and lifetime treatments, physical examination, laboratory screening, transthoracic Doppler echocardiography, and the six-minute walk test. Blood pressure was obtained according to the standard method at each site with the cuff placed in the forearm and the patient seated. Routine laboratory tests including complete blood count, serum chemistry profile, lactate dehydrogenase (LDH), urinalysis, urine albumin, and urine creatinine from samples taken at the subject's screening visit were performed in the local laboratories of the participating institutions. Echocardiography was performed at the participating institutions and read centrally in the National Heart, Lung and Blood Institute echocardiography core laboratory. Cardiac measurements were performed according to American Society of Echocardiography guidelines.[25] Percentage of hemoglobin F was measured by high-performance liquid chromatography (Ultra Resolution System, Trinity Biotech, Jamestown, NY). Alpha-thalassemia was detected by molecular methodology based on polymerase chain reaction at the University of Pittsburgh. Serum N-terminal pro-brain natriuretic peptide (NT-pro BNP) concentration was measured by a sandwich immunoassay using polyclonal antibodies that recognize epitopes located in the N-terminal segment (1-76) of pro-BNP (1-108) (Elecsys analyser; Roche Diagnostics, Mannheim, Germany). Ferritin was measured with an enzyme immunoassay (Ramco Laboratories Inc., Stafford, TX). The eGFR was calculated using the CKD-EPI formula (eGFR in $\mathrm{ml} / \mathrm{min} /$ $1.73 \mathrm{~m}^{2}=175 \times(\text { serum creatinine })^{-1.154} \times(\text { age })^{-0.203} \times(0.742$ if female $) \times$ (1.212 if African American)).

\section{Statistical Analysis}

Patient characteristics are presented as median and interquartile range (IQR) or number and percentage of participants with a given characteristic, and associations with hemoglobin genotype were assessed using the Wilcoxon twosample test and the Pearson chi-square test of independence. A hemolytic component variable was derived using principal component analysis from four markers of hemolysis - LDH, aspartate aminotransferase (AST), total bilirubin, and reticulocyte percent - as described elsewhere.[24] This variable produced by data reduction methodology has strong relationships with indirect and direct measures of hemolysis, including cell-free hemoglobin levels and red blood cell microparticle counts.[12] Proteinuria was defined as any positive result from the urine dipstick test from urine samples taken at the subject's screening visit. Urine 
albumin and creatinine were available for a subset of subjects and were measured from spot urine samples taken at the screening visit. Chronic kidney disease (CKD) was defined according to the National Kidney Foundation, Kidney Disease Outcomes Quality Initiatives (K/DOQI) guidelines (National Kidney Foundation, 2002) to align the definition of CKD stage to the current evidence-based guidelines: stage $0-\mathrm{eGFR}>60 \mathrm{ml} / \mathrm{min} / 1.73 \mathrm{~m}^{2}$ and albuminuria $<30 \mathrm{mg} / \mathrm{g}$ creatinine; stage 1 - eGFR $\geqslant 90 \mathrm{ml} / \mathrm{min} / 1.73 \mathrm{~m}^{2}$ and albuminuria $\geqslant 30 \mathrm{mg} / \mathrm{g}$ creatinine; stage 2 - eGFR $60-89 \mathrm{ml} / \mathrm{min} / 1.73 \mathrm{~m}^{2}$ and albuminuria $\geqslant 30 \mathrm{mg} / \mathrm{g}$ creatinine; stage 3 - eGFR $<60 \mathrm{ml} / \mathrm{min} / 1.73 \mathrm{~m}^{2}$.

Associations of patient characteristics with pulse pressure were assessed using Spearman correlation coefficients and linear regression analysis, log-transforming continuous variables as necessary to normalize skewed distributions. Regression coefficients were tested for significant differences from zero by the t-test. For the final models, variables were entered in a stepwise approach if they had a significant univariate association with pulse pressure. All statistical analyses were performed using SAS, version 9.1 (SAS Institute, Inc., Cary, NC) and Stata, version 11.1 (Statacorp, LP, College Station, TX).

\section{Results}

\section{Patient characteristics by hemoglobin genotype}

Most patients enrolled in walk-PHaSST had either HbSS or HbSC and were analyzed separately. We are reporting their baseline characteristics as these are not available from prior walk-PHaSST published reports (Table 1). As expected based on prior epidemiological studies, we found significant differences among subjects with $\mathrm{HbSS}$ and HbSC disease. HbSS patients were significantly younger and had lower BMI and oxygen saturation than patients with HbSC disease. Among the blood pressure parameters, pulse pressure was the only one higher in the HbSS group, while systolic, diastolic and mean arterial pressures were all significantly higher in HbSC patients. The prevalence of patients on hydroxyurea was higher in the HbSS group as compared to the HbSC group, which resulted in higher fetal hemoglobin levels. There was also a higher proportion of patients on chronic transfusion in the HbSS group, which was accompanied by a higher ferritin value in this group. HbSS patients had higher markers of hemolysis as measured by reticulocyte proportion, $\mathrm{LDH}, \mathrm{AST}$, total and hemolytic component, all validated by specific measures of cell free plasma hemoglobin and red cell derived microparticles.[12] As a result of the higher hemolysis, the total hemoglobin level was lower in patients with HbSS than in patients with HbSC. A larger proportion of HbSS patients had proteinuria, a marker of sickle cell nephropathy, recently shown to be predicted by hemolysis and hemoglobinuria,[26] and chronic kidney disease stage I or higher. In keeping with these findings, they had a higher estimated glomerular filtration rate, indicative of worse glomerular hyperfiltration. Finally, the surrogate markers of pulmonary hypertension TRV and NTproBNP were significantly different among the two genotypes. 
Table 1. Patient characteristics by sickle cell disease genotype.

\begin{tabular}{|c|c|c|c|c|c|}
\hline \multirow[t]{2}{*}{ Patient characteristics } & \multicolumn{2}{|c|}{ HbSS patients } & \multicolumn{2}{|c|}{ HbSC patients } & \multirow[b]{2}{*}{$\mathbf{p}^{\dagger}$} \\
\hline & $n$ & median (IQR)* & 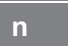 & median (IQR)* & \\
\hline Age, years & 500 & $34(25-45)$ & 161 & $41(28-51)$ & 0.002 \\
\hline Male gender, $\mathrm{N}(\%)$ & 500 & $245(49.0)$ & 161 & $66(41.0)$ & 0.08 \\
\hline $\mathrm{SBP}, \mathrm{mmHg}$ & 500 & $117(109-127)$ & 161 & $120(112-132)$ & 0.01 \\
\hline $\mathrm{DBP}, \mathrm{mmHg}$ & 500 & $67(60-74)$ & 161 & $71(67-80)$ & $<0.0001$ \\
\hline MAP, $\mathrm{mmHg}$ & 500 & $83(77-90)$ & 161 & $88(82-96)$ & $<0.0001$ \\
\hline Pulse pressure, $\mathrm{mmHg}$ & 500 & $50(42-59)$ & 161 & $48(41-55)$ & 0.02 \\
\hline Body mass index (BMI) & 492 & $23.0(20.8-25.8)$ & 160 & $26.7(22.7-30.5)$ & $<0.0001$ \\
\hline Oxygen saturation $\%$ & 497 & 97 (95-98) & 160 & 98 (97-99) & $<0.0001$ \\
\hline Hydroxyurea use, N (\%) & 500 & $225(45.0)$ & 161 & $21(13.0)$ & $<0.0001$ \\
\hline Transfusions ${ }^{\S}, \mathrm{N}(\%)$ & 499 & $72(14.4)$ & 159 & $6(3.8)$ & 0.0003 \\
\hline Chronic pain, $\mathrm{N}(\%)$ & 499 & $184(36.9)$ & 161 & $78(48.4)$ & 0.009 \\
\hline Six minute walk, $m$ & 492 & $438(384-504)$ & 159 & $440(363-495)$ & 0.32 \\
\hline WBC, $\times 10^{9} / \mathrm{L}$ & 490 & $9.7(7.5-4.3)$ & 156 & $8.1(6.4-10.0)$ & $<0.0001$ \\
\hline Hemoglobin, mmol/L & 489 & $5.4(4.7-6.1)$ & 157 & $6.9(6.4-7.6)$ & $<0.0001$ \\
\hline Hematocrit, \% & 490 & $25.2(22.0-28.4)$ & 157 & $32.4(29.4-35.3)$ & $<0.0001$ \\
\hline Platelets, $\times 10^{9} / \mathrm{L}$ & 489 & $364(285-456)$ & 156 & $296(193-362)$ & $<0.0001$ \\
\hline Hemoglobin F, \% & 445 & $6.3(2.6-12.4)$ & 139 & $1.5(0.8-3.2)$ & $<0.0001$ \\
\hline Reticulocytes, \% & 461 & $9.1(6.1-13.4)$ & 153 & $3.3(2.5-4.7)$ & $<0.0001$ \\
\hline LDH, IU/L & 453 & $422(299-600)$ & 154 & $240(200-321)$ & $<0.0001$ \\
\hline Total Bilirubin, $\mu \mathrm{mol} / \mathrm{L}$ & 490 & $46.2(32.5-70.1)$ & 159 & $22.2(15.4-34.2)$ & $<0.0001$ \\
\hline Hemolytic Component & 420 & $0.6(-0.4-1.4)$ & 148 & $-1.6(-2.3-1.0)$ & $<0.0001$ \\
\hline AST, IU/L & 477 & $42(31-59)$ & 157 & $26(21-35)$ & $<0.0001$ \\
\hline Ferritin, pmol/L & 450 & 643 (254-1539) & 141 & $263(148-483)$ & $<0.0001$ \\
\hline Albumin, $g / L$ & 489 & $42(39-44)$ & 157 & $42(39-44)$ & 0.59 \\
\hline ALT, IU/L & 491 & $23(17-33)$ & 159 & $18(14-26)$ & $<0.0001$ \\
\hline ALP, IU/L & 489 & $90(70-122)$ & 157 & $76(65-102)$ & 0.0002 \\
\hline Creatinine, $\mu \mathrm{mol} / \mathrm{L}$ & 492 & $61.9(50.4-79.6)$ & 159 & 70.7 (61.9-86.6) & $<0.0001$ \\
\hline Proteinuria, N (\%) & 474 & $162(34.2)$ & 147 & $28(19.0)$ & 0.0005 \\
\hline ACR & 306 & $32(7.3-220.0)$ & 81 & $15.2(4.2-68.4)$ & 0.004 \\
\hline eGFR $\left(\mathrm{ml} / \mathrm{min} / 1.73 \mathrm{~m}^{2}\right)$ & 492 & $138(109-153)$ & 157 & $119(95-137)$ & $<0.0001$ \\
\hline CKD", N (\%) & 313 & $173(55.3)$ & 82 & $35(42.7)$ & 0.04 \\
\hline TRV, m/sec & 449 & $2.6(2.3-2.7)$ & 139 & $2.4(2.2-2.7)$ & 0.003 \\
\hline NT-proBNP, pmol/L & 464 & $9.1(4.0-19.9)$ & 146 & $5.3(2.2-12.5)$ & $<0.0001$ \\
\hline
\end{tabular}

*Unless otherwise indicated; ${ }^{\dagger}$ From Wilcoxon two-sample test for difference in medians or Pearson chi-square test of independence of groups. $p$ values $<0.002$ remained significant after Bonferroni's adjustment for multiple comparisons; "Hemoglobin oxygen saturation; ${ }^{\S}$ Chronic transfusion therapy; "Stage I or higher.

$\mathrm{SBP}=$ systolic blood pressure; $\mathrm{DBP}=$ diastolic blood pressure; $\mathrm{MAP}=$ mean arterial pressure; $\mathrm{WBC}=$ white blood cell count; $\mathrm{LDH}=$ lactate dehydrogenase; $\mathrm{AST}=$ aspartate aminotransferase; $\mathrm{ALT}=$ alanine aminotransferase; $\mathrm{ALP}=$ alkaline phosphatase; $\mathrm{ACR}=$ urine albumin-to-creatinine ratio; eGFR=estimated glomerular filtration rate; $\mathrm{CKD}=$ chronic kidney disease; $\mathrm{TRV}=$ tricuspid regurgitant jet velocity; $\mathrm{NT}$-proBNP= $\mathrm{N}$-terminal prohormone of brain natriuretic peptide.

doi:10.1371/journal.pone.0114309.t001 
Elevated pulse pressure correlates with markers of hemolysis, elevated creatinine and proteinuria in HbSS patients

We next conducted a univariate analysis of pulse pressure and multiple clinical and laboratory markers in patients with HbSS and HbSC disease (Table 2). In HbSS patients, a high pulse pressure was associated with male gender and was negatively correlated with hemoglobin oxygen saturation. We also found that pulse pressure was consistently and positively correlated with all markers of hemolysis, including reticulocyte proportion, LDH, total bilirubin, AST and hemolytic component (Figure 1, panel A). Finally, pulse pressure was positively correlated with creatinine (Figure 1, panel B), urine albumin-to-creatinine ratio and presence of proteinuria (Figure 1, panel C).

In HbSC patients, a different pattern of pulse pressure correlates emerged. In this group, pulse pressure was positively correlated with age and BMI. There was no correlation with markers of hemolysis and although, similarly to HbSS patients, there was a positive correlation with creatinine (Figure 1, panel B), there was no significant correlation with presence of proteinuria or the urine albuminto-creatinine ratio.

In both HbSS and HbSC groups pulse pressure was positively correlated with TRV.

A complete list of all univariate correlations is reported in Table 2.

\section{Pulse pressure is the only systemic pressure parameter associated with hemolysis by multiple linear regression in $\mathrm{HbSS}$ patients}

We conducted a multiple linear regression analysis to determine which of the variables that emerged as significant by univariate analysis were able to crosssectionally predict the blood pressure variables (systolic, diastolic, mean arterial and pulse pressure) in the regression model. The following pairs of variables could not be entered into the model simultaneously due to their high correlation, so we fitted separate models for each of them: reticulocytes and hemolytic component; creatinine and estimated glomerular filtration rate; and proteinuria and urine albumin-to-creatinine ratio. For brevity, we are only reporting the results of the regression analysis for pulse pressure for HbSS and HbSC patients, separately.

As shown in Table 3, pulse pressure was predicted by hemolysis in HbSS patients, while systolic, diastolic and mean arterial pressure were not (data not shown).

In model 1, where the hemolysis markers LDH, AST, total bilirubin, and reticulocyte proportion were coded as individual variables, the reticulocyte proportion emerged as a predictor of pulse pressure, together with TRV $\geqslant 3.0 \mathrm{~m} /$ $\mathrm{sec}$, hemoglobin oxygen saturation, creatinine and proteinuria. In model 2 , the hemolytic component emerged as a significant predictor of pulse pressure in the regression model. Model 3 and 4 were fitted with the urine album-to-creatinine ratio replacing proteinuria and showed similar results. 
Table 2. Correlations of pulse pressure with clinical and laboratory characteristics by hemoglobin genotype.

\begin{tabular}{|c|c|c|c|c|}
\hline & \multicolumn{2}{|c|}{ HbSs patients } & \multicolumn{2}{|c|}{ HbSC patients } \\
\hline & $n$ & Spearman rho $(p)^{*}$ & $n$ & Spearman rho $(p)^{*}$ \\
\hline Age & 500 & $-0.005(0.91)$ & 161 & $0.18(0.03)$ \\
\hline Male gender, $\mathrm{N}(\%)$ & 500 & $0.15(0.0009)$ & 161 & $0.10(0.19)$ \\
\hline Systolic blood pressure & 500 & $0.66(<0.0001)$ & 161 & $0.73(<0.0001)$ \\
\hline Diastolic blood pressure & 500 & $-0.29(<0.0001)$ & 161 & $-0.06(0.46)$ \\
\hline Mean arterial pressure & 500 & $0.09(0.04)$ & 161 & $0.33(<0.0001)$ \\
\hline $\mathrm{BMI}$ & 492 & $-0.02(0.64)$ & 160 & $0.26(0.001)$ \\
\hline Hemoglobin oxygen saturation & 497 & $-0.17(0.0001)$ & 160 & $-0.10(0.22)$ \\
\hline Hydroxyurea, current use, $\mathrm{N}(\%)$ & 500 & $-0.02(0.70)$ & 161 & $0.009(0.91)$ \\
\hline Chronic transfusion therapy, $\mathrm{N}(\%)$ & 499 & $-0.002(0.96)$ & 159 & $-0.02(0.80)$ \\
\hline Chronic pain, $\mathrm{N}(\%)$ & 499 & $-0.03(0.47)$ & 161 & $-0.06(0.47)$ \\
\hline Six minute walk, $\mathrm{m}$ & 492 & $0.09(0.04)$ & 159 & $0.03(0.71)$ \\
\hline White blood cells & 490 & $0.09(0.04)$ & 156 & $0.11(0.15)$ \\
\hline Hemoglobin & 489 & $-0.06(0.17)$ & 157 & $-0.009(0.91)$ \\
\hline Hematocrit & 490 & $-0.07(0.13)$ & 157 & $0.02(0.79)$ \\
\hline Platelets & 489 & $0.02(0.61)$ & 156 & $0.02(0.81)$ \\
\hline Hemoglobin $\mathrm{F}$ & 445 & $-0.01(0.81)$ & 139 & $0.02(0.81)$ \\
\hline Reticulocytes & 461 & $0.16(0.0006)$ & 153 & $0.10(0.20)$ \\
\hline Lactate dehydrogenase & 453 & $0.15(0.001)$ & 154 & $0.07(0.41)$ \\
\hline Total Bilirubin & 490 & $0.11(0.02)$ & 159 & $0.001(0.99)$ \\
\hline Hemolytic Component & 420 & $0.21(<0.0001)$ & 148 & $0.09(0.28)$ \\
\hline Aspartate aminotransferase & 477 & $0.13(0.006)$ & 157 & $0.13(0.12)$ \\
\hline Ferritin & 450 & $-0.05(0.28)$ & 141 & $0.22(0.008)$ \\
\hline Albumin & 489 & $-0.09(0.06)$ & 157 & $-0.05(0.54)$ \\
\hline Alanine aminotransferase & 491 & $0.08(0.07)$ & 159 & $0.20(0.01)$ \\
\hline Alkaline Phosphatase & 489 & $0.09(0.04)$ & 157 & $0.03(0.68)$ \\
\hline Creatinine & 492 & $0.10(0.02)$ & 159 & $0.28(0.0004)$ \\
\hline Protein in urine & 474 & $0.16(0.0005)$ & 147 & $0.02(0.82)$ \\
\hline Urine album-to-creatinine ratio & 306 & $0.15(0.01)$ & 81 & $0.20(0.08)$ \\
\hline Estimated glomerular filtration rate & 492 & $-0.006(0.9)$ & 157 & $-0.25(0.002)$ \\
\hline Chronic kidney disease & 313 & $0.16(0.006)$ & 82 & $0.23(0.04)$ \\
\hline Tricuspid regurgitant jet velocity & 449 & $0.20(<0.0001)$ & 139 & $0.20(0.02)$ \\
\hline NT-proBNP ${ }^{\dagger}$ & 464 & $0.09(0.06)$ & 146 & $0.12(0.16)$ \\
\hline
\end{tabular}

${ }^{*} p$ values $<0.002$ remained significant after Bonferroni's adjustment for multiple comparisons.

$\mathrm{N}$-terminal prohormone of brain natriuretic peptide.

doi:10.1371/journal.pone.0114309.t002

In hemoglobin SC patients, creatinine (model 1) and estimated glomerular filtration rate (model 2) and ferritin (both models) were the only predictors of pulse pressure (Table 3 ). 
A

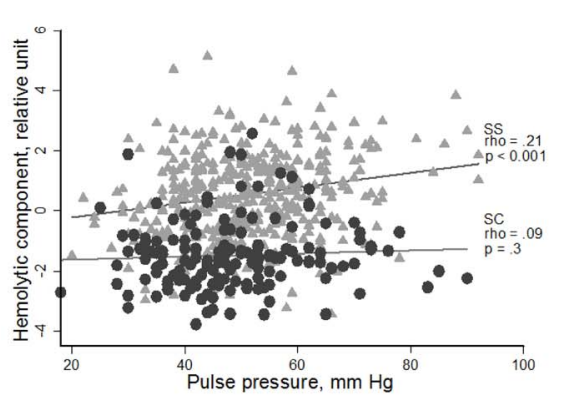

B

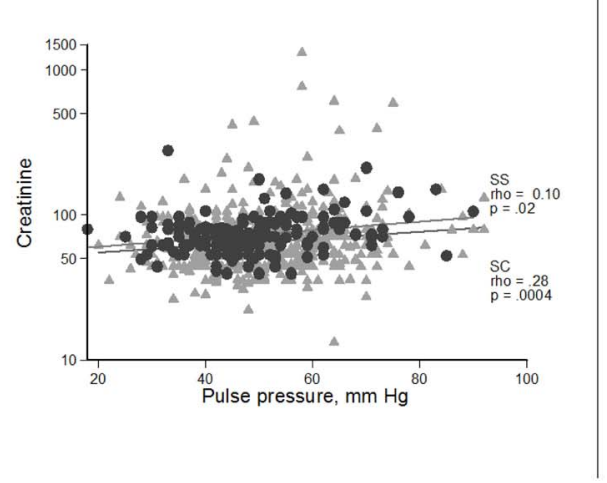

C

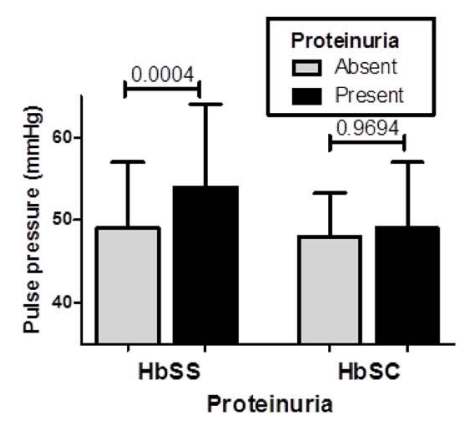

Figure 1. Correlates and associations of pulse pressure with kidney function and hemolysis. A, Pulse pressure has a significant positive correlation with the hemolytic component in HbSS patients, but not in HbSC patients. B, Pulse pressure has a significant positive correlation with serum creatinine in both $\mathrm{HbSS}$ and $\mathrm{HbSC}$ patients. C, Elevated pulse pressure is significantly associated with presence of proteinuria in $\mathrm{HbSS}$ patients, while the association is not significant in $\mathrm{HbSC}$ patients.

doi:10.1371/journal.pone.0114309.g001

\section{Discussion}

Pulse pressure is emerging as an important risk factor for end-organ damage and cardiovascular morbidity and mortality in many conditions (reviewed in [18]). While several studies have explored the associations of systolic blood pressure in SCD, the role of pulse pressure has never been thoroughly investigated in this disease.

Our study shows that pulse pressure is predicted by hemolysis in patients with HbSS from the walk-PHaSST international multi-center cross-sectional cohort. To our knowledge, this is the largest SCD cohort since the CSSCD, a natural history study that followed SCD patients in the pre-hydroxyurea era. A striking finding of the CSSCD was that SCD patients, and particularly those with HbSS had lower overall median systolic blood pressure $(113 \pm 14.5 \mathrm{mmHg})$ than normative controls, a difference observed for all age groups and both sexes.[1] In the hydroxyurea era, the UNC cohort was characterized by a higher systolic blood pressure $(122 \pm 15 \mathrm{mmHg})$ which was not significantly different from that of age-matched African American control subjects.[5] Both and other studies have shown that elevations of systolic blood pressure over baseline are detrimental in SCD patients and predict a risk of vascular complications including stroke, $[1,27]$ kidney disease, $[28,29]$ and pulmonary hypertension. [24] It is, however, intriguing that while high baseline hemolysis is independently correlated with these complications, no association had been found between systolic blood pressure and severity of hemolysis.[] ] Our findings of a lack of association between markers of hemolysis and systolic blood pressure in multiple regression analysis in the walkPHaSST cohort confirm these prior reports. We, therefore, hypothesized that the detrimental effect of systolic blood pressure may be mediated by pulse pressure elevation in response to hemolysis-derived vasculopathy. To this day, little 
Table 3. Independent predictors of pulse pressure.

Beta $(95 \% \mathrm{Cl})$

\section{HbSS patients}

Model $1(n=387)$

Reticulocytes*, \%

TRV, m/sec

Hemoglobin oxygen saturation

Creatinine*

Proteinuria

Model $2(n=352)$

Hemolytic component

TRV, m/sec

Hemoglobin oxygen saturation

Creatinine*

Proteinuria

Model $3(n=281)$

Reticulocytes*, \%

Urine albumin-to-creatinine ratio*

Creatinine*

Hemolytic component

Urine albumin-to-creatinine ratio*

Creatinine*

\section{HbSC patients}

Model $1(n=140)$

Creatinine*

Ferritin*

eGFR

Ferritin $^{*}$
Model $4(n=263)$

Model $2(n=138)$

\begin{tabular}{|c|c|c|}
\hline $2.07(0.1-4.0)$ & 0.04 & 0.11 \\
\hline $13.03(5.2-20.9)$ & 0.001 & 0.17 \\
\hline$-0.42(-0.79-0.05)$ & 0.03 & -0.12 \\
\hline $2.88(0.19-5.58)$ & 0.04 & 0.11 \\
\hline $2.58(0.20-4.97)$ & 0.03 & 0.11 \\
\hline $1.37(0.4-2.3)$ & 0.006 & 0.16 \\
\hline $10.04(1.7-18.4)$ & 0.02 & 0.13 \\
\hline$-0.34(-0.7-0.05)$ & 0.09 & -0.10 \\
\hline $3.34(0.6-6.1)$ & 0.02 & 0.13 \\
\hline $2.57(0.08-5.0)$ & 0.04 & 0.11 \\
\hline $2.42(0.3-4.6)$ & 0.03 & 0.13 \\
\hline $0.64(0.01-1.3)$ & 0.05 & 0.12 \\
\hline $3.22(0.2-6.2)$ & 0.03 & 0.13 \\
\hline $1.50(0.4-2.6)$ & 0.006 & 0.17 \\
\hline $0.56(-0.1-1.2)$ & 0.09 & 0.11 \\
\hline $4.00(0.8-7.2)$ & 0.01 & 0.16 \\
\hline $9.65(3.0-16.3)$ & 0.005 & 0.23 \\
\hline $2.66(0.7-4.6)$ & 0.008 & 0.22 \\
\hline$-0.08(-0.14,-0.02)$ & 0.01 & -0.21 \\
\hline $2.63(0.7-4.6)$ & 0.009 & 0.22 \\
\hline
\end{tabular}

*transformed using the natural log function.

doi:10.1371/journal.pone.0114309.t003 attention has been devoted to pulse pressure in SCD, an increasingly recognized cardiovascular risk factor. While the mechanistic link between hemolysis, pulse pressure and vascular complications cannot be proven in an epidemiological cross-sectional study, our findings do suggest that elevated pulse pressure may be more reflective of the peculiar vasculopathy of SCD, where hemolysis causes increased arterial stiffness from nitric oxide depletion and decreased peripheral vascular resistance from anemia, leading to elevated pulse pressure. Moreover, hemolysis is also linked to microvascular dysfunction in SCD, since the primary pathogenic process in this disease occurs at the level of the post-capillary venules and is characterized by cellular adhesion, vaso-occlusion, hemolysis and ischemiareperfusion injury.[30] Microvascular damage would lead to loss of small vessels and consequent increased arterial stiffness which would elevate pulse pressure. 
Elevated pulse pressure would in turn damage small vessels and lead to microvascular rarefaction in a vicious cycle. $[18,31]$ It is, therefore, possible that hemolysis may mechanistically link both micro and microvascular dysfunction and that elevated pulse pressure may be a result of cross-talk between large and small caliber vessels as documented in hypertensive patients.[18] The link between hemolysis and pulse pressure is also supported by our finding that hemolysis was not associated with pulse pressure in HbSC, a subgroup of SCD which is not characterized by high level hemolysis and whose disease manifestations may result, instead, from increased blood viscosity.

As other studies have shown, $[\underline{5}, \underline{32}]$ we found a strong correlation between all pressure parameters and kidney function measured as serum creatinine and proteinuria. This finding reinforces the importance of systemic blood pressure as a predictor of kidney deterioration in SCD. Since abnormalities in kidney function are among the most sensitive and earliest biomarkers of microvascular dysfunction in SCD,[33] it may be important to therapeutically target blood pressure early and aggressively, to prevent this complication. While angiotensinconverting enzyme inhibitors may reduce proteinuria in SCD patients, [34] there is no clear evidence to show which patients may benefit the most from these medications. In particular, it is not known what the optimal blood pressure threshold to initiate angiotensin-converting enzyme inhibitor therapy should be and whether reduction of albuminuria predicts long term clinical outcomes such as need for renal replacement therapy and death. Based on our results, treatments aimed at reducing hemolysis may, however, also benefit the kidney microvascular district, independently from other therapeutic pathways.

We also found a significant association between BMI and pulse pressure in HbSC patients, but not in HbSS patients. In HbSC patients, we also observed an interaction of gender and BMI, with associations of BMI with pulse pressure observed in males but not in females (data not shown). BMI has been positively correlated with pulse pressure in other populations without SCD, [35] thus suggesting that in older HbSC patients with increased BMI, obesity may have the same adverse impact on arterial compliance observed in populations exposed to routine cardiovascular risk factors. It is intriguing to hypothesize that other comorbid conditions such as dyslipidemia and diabetes may mediate the association between BMI and pulse pressure in HbSC patients.

Finally, we found significant independent associations between hemoglobin oxygen saturation and pulse pressure in HbSS patients and ferritin and pulse pressure in HbSC patients. We hypothesize that the inverse relationship between hemoglobin oxygen saturation and pulse pressure in HbSS patients may be either the result of increased cardiopulmonary disease burden in patients with high pulse pressure, as also shown by the association with elevated TRV, or worse anemia secondary to hemolysis. As shown in Table 1, patients with HbSC had significantly lower ferritin than HbSS patients with the IQ range below 500 pmol/L. Patients with HbSC have significantly lower burden of hemosiderosis as compared to HbSS patients as they are less frequently transfused due to higher baseline hemoglobin. Thus, values in the range observed in this study may be more 
reflective of an enhanced inflammatory state. If this hypothesis is correct, pulse pressure may be positively correlated with increased inflammation in $\mathrm{HbSC}$ patients from our cohort.

The main limitation of our study is that the walk-PHaSST clinical cohort was not designed to specifically evaluate baseline systemic blood pressure in SCD patients. Thus, data on the history of hypertension or use of antihypertensive medications are not available for our population. The use of principal components analysis to derive a hemolytic component variable from several correlated variables all related to hemolysis is an additional limitation because of potentially important associations that could have been missed by using a data reduction technique such as PCA. Finally, eGFR and creatinine are relatively poor markers of kidney function in sickle cell disease due to glomerular hyperfiltration and increased creatinine excretion by the proximal tubule in this disease. It is, unfortunately, not possible to infer which patient subsets may have experienced worse hyperfiltration and the confounding effect of this phenomenon, although it is safe to assume that glomerular hyperfiltration decreased with increasing age in our population. Associations of eGFR with pulse pressure did not, however, change after adjustment for age. Our results are also strengthened by the availability of values of proteinuria and albumin to creatinine ratio in a subset of patients. These are recognized valuable screening tools of kidney dysfunction by the recently published sickle cell guidelines.[36]

In summary, our results show that pulse pressure is closely linked to hemolysis in SCD and suggest that it may be an important predictor of cardiovascular risk. Longitudinal and mechanistic studies should be conducted to confirm these hypotheses. Moreover, measurement of pulse wave velocity may provide further information on arterial stiffness and its relationship to pulse pressure and microvascular dysfunction in SCD.

\section{Acknowledgments}

We thank the walk-PHaSST investigators who are not co-authors and patients for biobank and database contribution to this study.

\section{Author Contributions}

Conceived and designed the experiments: EMN MH MTG. Performed the experiments: EMN MH MTG. Analyzed the data: EMN MH. Wrote the paper: EMN MH CR RV MS GJK MTG.

\section{References}

1. Pegelow CH, Colangelo L, Steinberg M, Wright EC, Smith J, et al. (1997) Natural history of blood pressure in sickle cell disease: risks for stroke and death associated with relative hypertension in sickle cell anemia. American Journal of Medicine 102: 171-177. 
2. Johnson CS, Giorgio AJ (1981) Arterial blood pressure in adults with sickle cell disease. Arch Intern Med 141: 891-893.

3. Rodgers GP, Walker EC, Podgor MJ (1993) Is "relative" hypertension a risk factor for vaso-occlusive complications in sickle cell disease? Am J Med Sci 305: 150-156.

4. Chien S, Usami S, Bertles JF (1970) Abnormal rheology of oxygenated blood in sickle cell anemia. J Clin Invest 49: 623-634.

5. Desai PC, Deal AM, Brittain JE, Jones S, Hinderliter A, et al. (2012) Decades after the cooperative study: a re-examination of systemic blood pressure in sickle cell disease. American Journal of Hematology 87: E65-68.

6. Gladwin MT, Schechter AN, Ognibene FP, Coles WA, Reiter CD, et al. (2003) Divergent nitric oxide bioavailability in men and women with sickle cell disease. Circulation 107: 271-278.

7. Kaul DK, Liu XD, Chang HY, Nagel RL, Fabry ME (2004) Effect of fetal hemoglobin on microvascular regulation in sickle transgenic-knockout mice. J Clin Invest 114: 1136-1145.

8. Reiter CD, Wang X, Tanus-Santos JE, Hogg N, Cannon RO 3rd, et al. (2002) Cell-free hemoglobin limits nitric oxide bioavailability in sickle-cell disease. Nat Med 8: 1383-1389.

9. Belhassen L, Pelle G, Sediame S, Bachir D, Carville C, et al. (2001) Endothelial dysfunction in patients with sickle cell disease is related to selective impairment of shear stress-mediated vasodilation. Blood 97: 1584-1589.

10. Aderibigbe A, Omotoso AB, Awobusuyi JO, Akande TM (1999) Arterial blood pressure in adult Nigerian sickle cell anaemia patients. West Afr J Med 18: 114-118.

11. Homi J, Homi-Levee L, Gentles S, Thomas P, Serjeant G (1993) Adolescent blood pressure in a cohort study of sickle cell disease. Arch Intern Med 153: 1233-1236.

12. Nouraie M, Lee JS, Zhang Y, Kanias T, Zhao X, et al. (2013) The relationship between the severity of hemolysis, clinical manifestations and risk of death in 415 patients with sickle cell anemia in the US and Europe. Haematologica 98: 464-472.

13. Fernandez-Fresnedo G, Rodrigo E, de Francisco AL, de Castro SS, Castaneda O, et al. (2006) Role of pulse pressure on cardiovascular risk in chronic kidney disease patients. Journal of the American Society of Nephrology 17: S246-249.

14. Franklin SS (2001) Blood pressure and cardiovascular disease: what remains to be achieved? Journal of Hypertension - Supplement 19: S3-8.

15. Safar ME, Balkau B, Lange C, Protogerou AD, Czernichow S, et al. (2013) Hypertension and vascular dynamics in men and women with metabolic syndrome. J Am Coll Cardiol 61: 12-19.

16. Belizna C, Loufrani L, Ghali A, Lahary A, Primard E, et al. (2012) Arterial stiffness and stroke in sickle cell disease. Stroke 43: 1129-1130.

17. Benetos A, Thomas F, Joly L, Blacher J, Pannier B, et al. (2010) Pulse pressure amplification a mechanical biomarker of cardiovascular risk. J Am Coll Cardiol 55: 1032-1037.

18. Laurent S, Briet M, Boutouyrie P (2009) Large and small artery cross-talk and recent morbiditymortality trials in hypertension. Hypertension 54: 388-392.

19. Rosano C, Watson N, Chang Y, Newman AB, Aizenstein HJ, et al. (2013) Aortic pulse wave velocity predicts focal white matter hyperintensities in a biracial cohort of older adults. Hypertension 61: 160-165.

20. Minneci PC, Deans KJ, Zhi H, Yuen PS, Star RA, et al. (2005) Hemolysis-associated endothelial dysfunction mediated by accelerated NO inactivation by decompartmentalized oxyhemoglobin. J Clin Invest 115: 3409-3417.

21. Aslan M, Thornley-Brown D, Freeman BA (2000) Reactive species in sickle cell disease. Ann N Y Acad Sci 899: 375-391.

22. Morris CR, Suh JH, Hagar W, Larkin S, Bland DA, et al. (2008) Erythrocyte glutamine depletion, altered redox environment, and pulmonary hypertension in sickle cell disease. Blood 111: 402-410.

23. Machado RF, Barst RJ, Yovetich NA, Hassell KL, Kato GJ, et al. (2011) Hospitalization for pain in patients with sickle cell disease treated with sildenafil for elevated TRV and low exercise capacity. Blood 118: 855-864. 
24. Sachdev V, Kato GJ, Gibbs JS, Barst RJ, Machado RF, et al. (2011) Echocardiographic Markers of Elevated Pulmonary Pressure and Left Ventricular Diastolic Dysfunction Are Associated With Exercise Intolerance in Adults and Adolescents With Homozygous Sickle Cell Anemia in the United States and United Kingdom. Circulation.

25. Lang RM, Bierig M, Devereux RB, Flachskampf FA, Foster E, et al. (2005) Recommendations for chamber quantification: a report from the American Society of Echocardiography's Guidelines and Standards Committee and the Chamber Quantification Writing Group, developed in conjunction with the European Association of Echocardiography, a branch of the European Society of Cardiology. Journal of the American Society of Echocardiography 18: 1440-1463.

26. Saraf SL, Zhang X, Kanias T, Lash JP, Molokie RE, et al. (2014) Haemoglobinuria is associated with chronic kidney disease and its progression in patients with sickle cell anaemia. British Journal of Haematology 164: 729-739.

27. Ohene-Frempong K, Weiner SJ, Sleeper LA, Miller ST, Embury S, et al. (1998) Cerebrovascular accidents in sickle cell disease: rates and risk factors. Blood 91: 288-294.

28. Gordeuk VR, Sachdev V, Taylor JG, Gladwin MT, Kato G, et al. (2008) Relative systemic hypertension in patients with sickle cell disease is associated with risk of pulmonary hypertension and renal insufficiency. American Journal of Hematology 83: 15-18.

29. Thompson J, Reid M, Hambleton I, Serjeant GR (2007) Albuminuria and renal function in homozygous sickle cell disease: observations from a cohort study. Arch Intern Med 167: 701-708.

30. Kaul DK, Fabry ME, Nagel RL (1989) Microvascular sites and characteristics of sickle cell adhesion to vascular endothelium in shear flow conditions: pathophysiological implications. Proc Natl Acad Sci U S A 86: 3356-3360.

31. James MA, Watt PA, Potter JF, Thurston H, Swales JD (1995) Pulse pressure and resistance artery structure in the elderly. Hypertension 26: 301-306.

32. Foucan L, Salmi LR, Billy-Brissac R, Bourhis V, Bangou J (1995) [Arterial pressure and urinary excretion of albumin in adults with sickle cell disease]. Presse Med 24: 1428-1432.

33. Falk RJ, Scheinman J, Phillips G, Orringer E, Johnson A, et al. (1992) Prevalence and pathologic features of sickle cell nephropathy and response to inhibition of angiotensin-converting enzyme. N Engl J Med 326: 910-915.

34. Sasongko TH, Nagalla S, Ballas SK (2013) Angiotensin-converting enzyme (ACE) inhibitors for proteinuria and microalbuminuria in people with sickle cell disease. Cochrane Database of Systematic Reviews 3: CD009191.

35. Kwagyan J, Tabe CE, Xu S, Maqbool AR, Gordeuk VR, et al. (2005) The impact of body mass index on pulse pressure in obesity. Journal of Hypertension 23: 619-624.

36. Yawn BP, Buchanan GR, Afenyi-Annan AN, Ballas SK, Hassell KL, et al. (2014) Management of sickle cell disease: summary of the 2014 evidence-based report by expert panel members. JAMA 312: 1033-1048. 